\title{
Complex image method for calculating electric and magnetic fields produced by an auroral electrojet of finite length
}

\author{
R. Pirjola, A. Viljanen \\ Finnish Meteorological Institute, Geophysical Research Division P. O. Box 503, FIN-00101 Helsinki, Finland \\ Tel.: + 3589 19294652; fax: + 3589 19294603; e-mail: Risto.Pirjola@fmi.fi
}

Received: 20 October 1997 / Revised: 3 February 1998 / Accepted: 10 March 1998

\begin{abstract}
The electromagnetic field due to ionospheric currents has to be known when evaluating space weather effects at the earth's surface. Forecasting methods of these effects, which include geomagnetically induced currents in technological systems, are being developed. Such applications are time-critical, so the calculation techniques of the electromagnetic field have to be fast but still accurate. The contribution of secondary sources induced within the earth leads to complicated integral formulas for the field at the earth's surface with a timeconsuming computation. An approximate method of calculation based on replacing the earth contribution by an image source having mathematically a complex location results in closed-form expressions and in a much faster computation. In this paper we extend the complex image method (CIM) to the case of a more realistic electrojet system consisting of a horizontal line current filament with vertical currents at its ends above a layered earth. To be able to utilize previous CIM results, we prove that the current system can be replaced by a purely horizontal current distribution which is equivalent regarding the total (= primary + induced) magnetic field and the total horizontal electric field at the earth's surface. The latter result is new. Numerical calculations demonstrate that CIM is very accurate and several magnitudes faster than the exact conventional approach.
\end{abstract}

Key words. Electromagnetic theory ·

Geomagnetic induction ·

Auroral ionosphere

\section{Introduction}

During geomagnetic disturbances, the changing magnetic field gives rise to an electric field producing

Correspondence to: R. Pirjola currents and voltages in electrical conductors such as power transmission systems, pipelines, phone cables and railway systems (Lanzerotti and Gregori, 1986; Boteler et al., 1998). To estimate these geomagnetically induced currents (GICs) and to prevent the problems they may cause, the horizontal electric field should be known at the earth's surface.

Häkkinen and Pirjola (1986) presented a general model of a three-dimensional ionospheric current system consisting of a horizontal sheet current having an arbitrary density distribution, and of geomagnetic-fieldaligned currents having any (fixed) direction. They derived exact formulas for the electromagnetic field at the surface of a layered earth. However, the resulting numerical integration over two horizontal wave numbers is highly time consuming. Consequently, such a computation is impossible to be combined to any timecritical applications, like forecasting of GICs, which is a topic intensively discussed and investigated today (Petschek and Feero, 1997).

The calculation becomes much simpler and faster if the earth contribution can approximately be represented by an image of the primary ionospheric source. This technique usually requires that the source is monochromatic, i.e. a single frequency is considered, and then the depth of the image is complex (Bannister, 1986). The complex location can be regarded just as a mathematical concept. Therefore it is not necessary to interpret the location physically although it also has a certain physical content reflecting the depth of induced currents within the earth (Szarka and Fischer, 1989). The use of the complex image method (CIM) was suggested by Wait and Spies (1969), and studied recently by Boteler and Pirjola (1998). The previous works mostly concentrate on modelling the electrojet by an infinitely long line current, an idealization which may lead to incorrect conclusions, especially in connection with GIC studies (Viljanen, 1997).

Thomson and Weaver (1975) presented a more general formulation of CIM in which any horizontal ionospheric current distribution is acceptable. The true 
current system in the earth's near-space is not horizontal but field-aligned currents between the ionosphere and the magnetosphere are important and must be taken into account. However, the well-known theorem by Fukushima (1976) shows that the real three-dimensional current system consisting of a vertical (field-aligned) current and horizontal currents is equivalent to a purely horizontal ionospheric current distribution as far as the earth-surface magnetic field due to the currents is considered. It would be tempting to assume that the equivalence is also valid for the electric field, making Thomson and Weaver's CIM formulation applicable to the real situation. In this paper (Appendix) we prove that this is the case, i.e. the equivalence concerns the total horizontal $(=$ primary + induced $)$ electric field as well. In the case of the electric field the induction in the earth, which is not treated by Fukushima, plays a significant role. A laterally uniform conductivity structure of the earth is assumed here.

Consequently, by combining the new equivalence result with Thomson and Weaver's treatment this paper provides an extension to previous CIM works. The theory is discussed, and numerical calculations show that CIM results very accurately agree with exact computations based on Häkkinen and Pirjola (1986). The particular model discussed in this paper consists of an electrojet represented by a line current of finite length with vertical currents at its ends. An extension to more complicated current systems constructed of a set of horizontal finite-length filaments with vertical currents at the ends is straightforward.

Mogilatov (1996) has also shown that a radial current sheet can equivalently be replaced by a vertical electric dipole. Aiming at controlled-source geophysical prospecting, he only considers the simpler case in which the current sheet lies at the air-earth interface (see also Wait, 1997).

\section{Theory}

Let us consider a horizontal line current of a finite length $L$ at the height $h$ above the earth's surface (Fig. 1). Field-aligned currents at the ends ensure current continuity, and in auroral regions they can be well approximated by vertical currents (Amm, 1995). A line current is a simple model of an electrojet, which is rather an ionospheric current sheet with a complicated horizontal distribution. In principle, any current system can be constructed as a superposition of a set of simple "U"-shaped currents depicted in Fig. 1.

Layered-earth models are considered in this paper, which is a reasonable large-scale approximation relevant in particular to GIC investigations. Lateral inhomogeneities of the earth's conductivity would cause additional complications. The standard coordinate system used in this paper is also defined in Fig. 1. We assume that the time dependence is given by $\exp (i \omega t)$ thus considering a single frequency $\omega$.

To derive CIM for an electrojet shown in Fig. 1, it seems natural to consider the complex image of a vertical current and combine it with the result by Thomson and Weaver (1975). From the theoretical viewpoint it is essential that considering the horizontal and vertical parts separately both contain divergent currents that cause accumulation of charges. Only when the two parts are put together are the charges cancelled. Thomson and Weaver derive the following formula for the complex skin depth $p$ (with different notation):

$p=\frac{Z}{i \omega \mu_{0}}$,

where $Z$ is the plane wave surface impedance, $\omega$ is the angular frequency and $\mu_{0}$ is the vacuum permeability. A recursion formula for the surface impedance associated with a layered earth is given by Wait (1981, pp. 52-53). The plane wave assumption now means that the two spatial wave numbers should be set equal to zero in Wait's equations. A straightforward and simple derivation of Eq. (1) is presented by Boteler and Pirjola (1998) in the case of an infinitely long line current.

Since the surface impedance $Z$ is generally a function of a wave number, denoted by $b$ (or as indicated above a function of a two-dimensional wave number vector), $p$ defined by Eq. (1) also depends on $b$. For a plane wave field $b$ is equal to zero, but for other fields $b$ gets nonzero values. Consequently, the use of the plane wave surface impedance in Eq. (1) involves an approximation. It should be noted that $p$ expressed by Eq. (1) in the wave number domain equals the "inductive response function" introduced in the geoelectromagnetic induction literature (e.g. Schmucker, 1970).

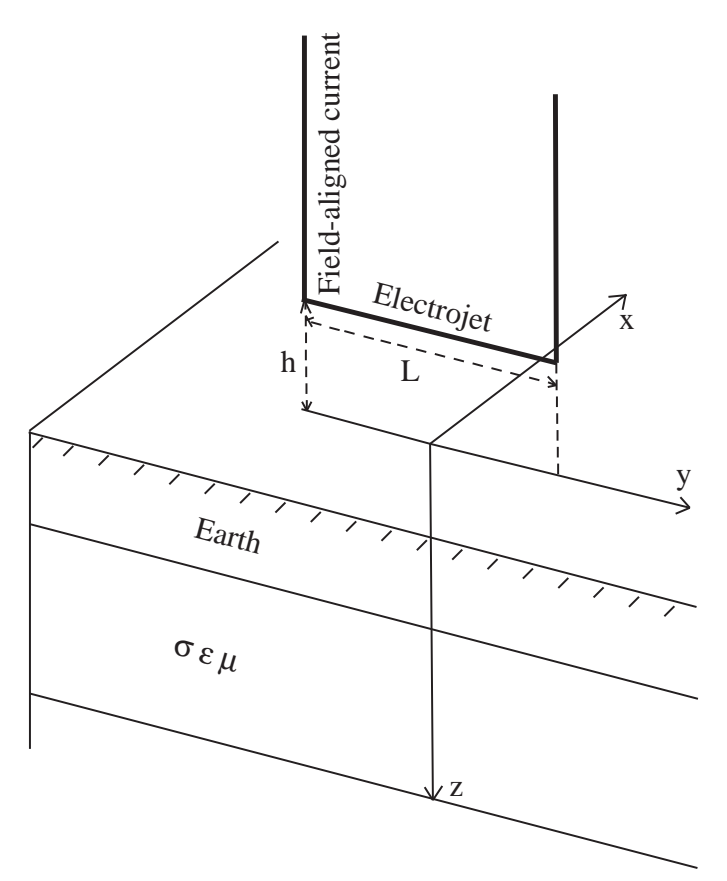

Fig. 1. Electrojet (amplitude $I$ ) of a finite length $L$ supplemented by vertical currents at the ends. The height of the electrojet is $h$, and the standard coordinate system with the earth's surface as the $x y$-plane, the $z$-axis downward and the $y$-axis parallel to the electrojet is used in this paper 
The image is situated at $z=h+2 p$ when the primary source lies at $z=-h$. Thomson and Weaver's treatment implicitly presumes that the horizontal ionospheric current is non-divergent. Therefore it is not clear whether Eq. (1) is also applicable to a divergent horizontal part of a more complicated electrojet system.

A vertical current is a superposition of successive vertical dipoles, which create an electromagnetic field expressible in terms of a Hertz vector (e.g. Wait, 1981, p. 16). Calculating the superposition integral and following exactly the same strategy with the surface impedance and the reflection coefficient as that used by Boteler and Pirjola (1998), we obtain the following formula for the complex skin depth implying the location of the starting point of the image current at $z=h+2 p$ :

$p=\frac{Z}{i \omega \mu_{0}} \frac{1}{1-\frac{b^{2}}{k_{0}^{2}}}$,

where $k_{0}$ is the propagation constant of the air given by Eq. (A2) (Appendix) with air parameters. Because $k_{0}$ is very small, usually approximated equal to zero in geomagnetic induction problems, the complex skin depths given by Eqs. (1) and (2) are not equal. Furthermore, the dependence of the right-hand side of Eq. (2) on the wave number $b$ makes the situation more complicated. All this indicates that the vertical image currents do not start exactly at the same complex points as the divergence points of the horizontal image currents. Consequently, a mere image current is not sufficient to describe the earth contribution in the case of a system consisting of horizontal and vertical currents, but image charges exist as well.

The existence of image charges can be understood by considering the "U"-shaped primary current shown in Fig. 1 as follows: the vertical electric field produced by the vertical currents causes charge accumulation at the interfaces between different media. The surface charges then create an electric field component parallel to the $x$ axis, i.e. perpendicular to the electrojet. If we now try to calculate the total field by using only two "U"-currents: the primary one and the secondary image current at a complex location, we will not obtain any electric $x$ component. This contradiction would obviously be avoided by also having image charges.

However, due to the inconvenient $b$-dependence appearing in Eq. (2), the location of the image charges is unclear and the simplicity involved in complex image formulations is lost. We will therefore proceed in another way with vertical currents. Considering the magnetic field at the earth's surface the vertical currents may be replaced by equivalent horizontal currents (Fukushima, 1976; Amm, 1997). (More precisely, the equivalence is only true with the accuracy of neglecting the displacement currents. Furthermore, these previous works do not explicitly take into account the effect of telluric currents.)

In the Appendix we consider a "U"-shaped current (Fig. 1) and show that it is equivalent with the horizontal system depicted in Fig. 2 regarding both the total $(=$ primary + induced $)$ magnetic and the total horizontal electric field at the earth's surface. It is worth noticing that the equivalence is also valid for the primary and induced magnetic fields separately, but as concerns the horizontal electric field, the equivalence only holds true for the total field.

Thus a realistic electrojet system containing vertical currents can be replaced by a purely horizontal current distribution (provided we are not concerned about the vertical electric field). This greatly simplifies the use of CIM since the formulation by Thomson and Weaver (1975) is then directly applicable.

In the Appendix, Eq. (A22) with the opposite sign, we show that the expression of the vector potential caused by a horizontal current distribution equivalent to a vertical current filament (amplitude $I$ ) flowing parallel to the $z$-axis down to the ionosphere at $z=-h$ is

$\boldsymbol{A}_{h}=-\frac{\mu_{0} I}{4 \pi} \frac{\rho}{\sqrt{\rho^{2}+(z+h)^{2}}+|z+h|} \hat{\boldsymbol{e}}_{\rho}$.

The vector potential due to the downward vertical current is (Eq. A17)

$\boldsymbol{A}_{v}=-\frac{\mu_{0} I}{4 \pi} \log \left(\sqrt{\rho^{2}+(z+h)^{2}}+(z+h)\right) \hat{\boldsymbol{e}}_{z}$.

The secondary field produced by the image is obtained by changing the sign of $\boldsymbol{A}_{\boldsymbol{h}}$ in Eq. (3) and replacing $h$ by $h+2 p$ where $p$ is given in Eq. (1). The field of a horizontal current filament can be similarly calculated in a closed form using Eqs. (A12)-(A14), and consequently the total field created by the "U"-current is obtainable.

The mathematical derivation presented by Thomson and Weaver (1975) shows that the CIM approximation is acceptable if the modulus of the complex depth is smaller than a characteristic horizontal changing size of the field, or alternatively very much larger. The former requirement is also included in the treatment by Boteler and Pirjola (1998). Wait and Spies (1969) regard the CIM formulation as valid provided the modulus of the complex skin depth in the earth is smaller than the distance between the source or its mirror image and the point of observation, which criterion is actually also mentioned by Thomson and Weaver. We have observed in practice that CIM works very well for typical parameter values in geomagnetic induction: period $10, \ldots, 1000 \mathrm{~s}$, earth resistivity $1, \ldots, 10^{5} \Omega \mathrm{m}$, height of ionospheric currents $100 \mathrm{~km}$, horizontal distance of the point of observation $0, \ldots, 1000 \mathrm{~km}$.

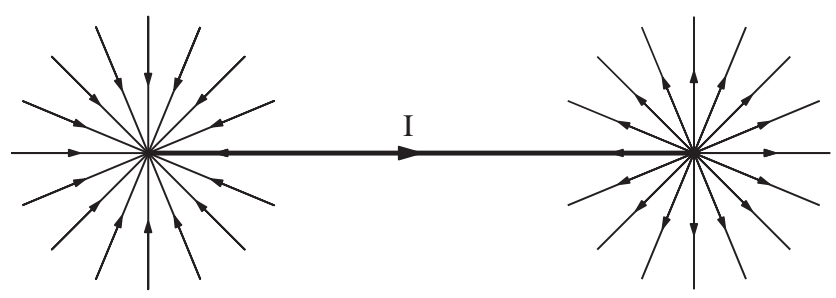

Fig. 2. Ionospheric current system equivalent to that in Fig. 1. The current is viewed from above 


\section{Numerical results}

We now compare the electromagnetic field calculated exactly and applying CIM. The case of an infinitely long line current has been investigated previously (e.g. Boteler and Pirjola, 1998), so we only discuss here an electrojet of a finite length presented in Fig. 1 and its equivalent model shown in Fig. 2.

The length, height and current strength of the electrojet are $L=1000 \mathrm{~km}, \quad h=110 \mathrm{~km}$ and $I=1000000 \mathrm{~A}$, and the period considered is $T=2 \pi / \omega=300 \mathrm{~s}$. The earth has three layers with thicknesses 20,30 and $\infty \mathrm{km}$, and with resistivities 100 , 2000 and $50 \Omega \mathrm{m}$. The earth is assumed to have the vacuum permeability $\mu_{0}$, and a permitivity of $5 \varepsilon_{0}$, although the latter does not affect the results in practice. The plane wave surface impedance now gets the value of about $0.0011+0.0015 i \Omega$, and so the complex skin depth obtained from Eq. (1) is $(57-42 i) \mathrm{km}$.

The exact calculations of the electric and magnetic fields at the earth's surface due to the current system depicted in Fig. 1 are based on the integral formulas by Häkkinen and Pirjola (1986). The Gauss Integration Formula is applied to the numerical computations (Pirjola and Häkkinen, 1991). The CPU time on a Unix work station (Silicon Graphics Power Challenge) required for an exact computation of the electromagnetic field on the given grid $(2000 \mathrm{~km} \times 2000 \mathrm{~km}$ with a $50-\mathrm{km}$ division) and at single frequency is in the order of 1 to $2 \mathrm{~h}$, while CIM based on the equivalent current distribution shown in Fig. 2 permits the calculation in a few seconds. (Utilizing specific routines applicable to some more restricted models may slightly decrease the difference of computer times.)

Figure 3 presents the three magnetic components and the two horizontal electric components as calculated exactly (solid lines) and using CIM (circles). The differences between the two methods are shown by the dashed lines. Figure 3 concerns an $x$ profile (perpendicular to the electrojet) at $y=100 \mathrm{~km}$. The results obtained exactly and applying CIM agree very well, so there is no question about the usefulness of CIM.

Figure 4 corresponds to Fig. 3 but the profile at which the fields are calculated lies at $y=500 \mathrm{~km}$, i.e. at the end of the electrojet. As can be seen, the accuracy of CIM is very good again. We have also considered more distant profiles up to $y=1000 \mathrm{~km}$ and found that the accuracy still holds true.

Figures 3 and 4 look rather similar, but it should be noted that the vertical scales are different. Near the centre of the electrojet at $y=100 \mathrm{~km} B_{x}$ and $B_{z}$ are much larger than $B_{y}$, and similarly $E_{y}$ dominates over $E_{x}$. However, near the end of the electrojet the importance of $B_{y}$ and $E_{x}$ increases. This emphasizes the fact that modelling an electrojet by an infinitely long line current, which completely neglects $B_{y}$ and $E_{x}$, may result in incorrect conclusions for example in GIC investigations.

It should also be noted that CIM leads to much more accurate results than letting the image lie at a real location determined by the assumption of a perfect conductor at a given depth within the earth (Lühr et al., 1984; Pirjola and Viljanen, 1989). With a real depth, the
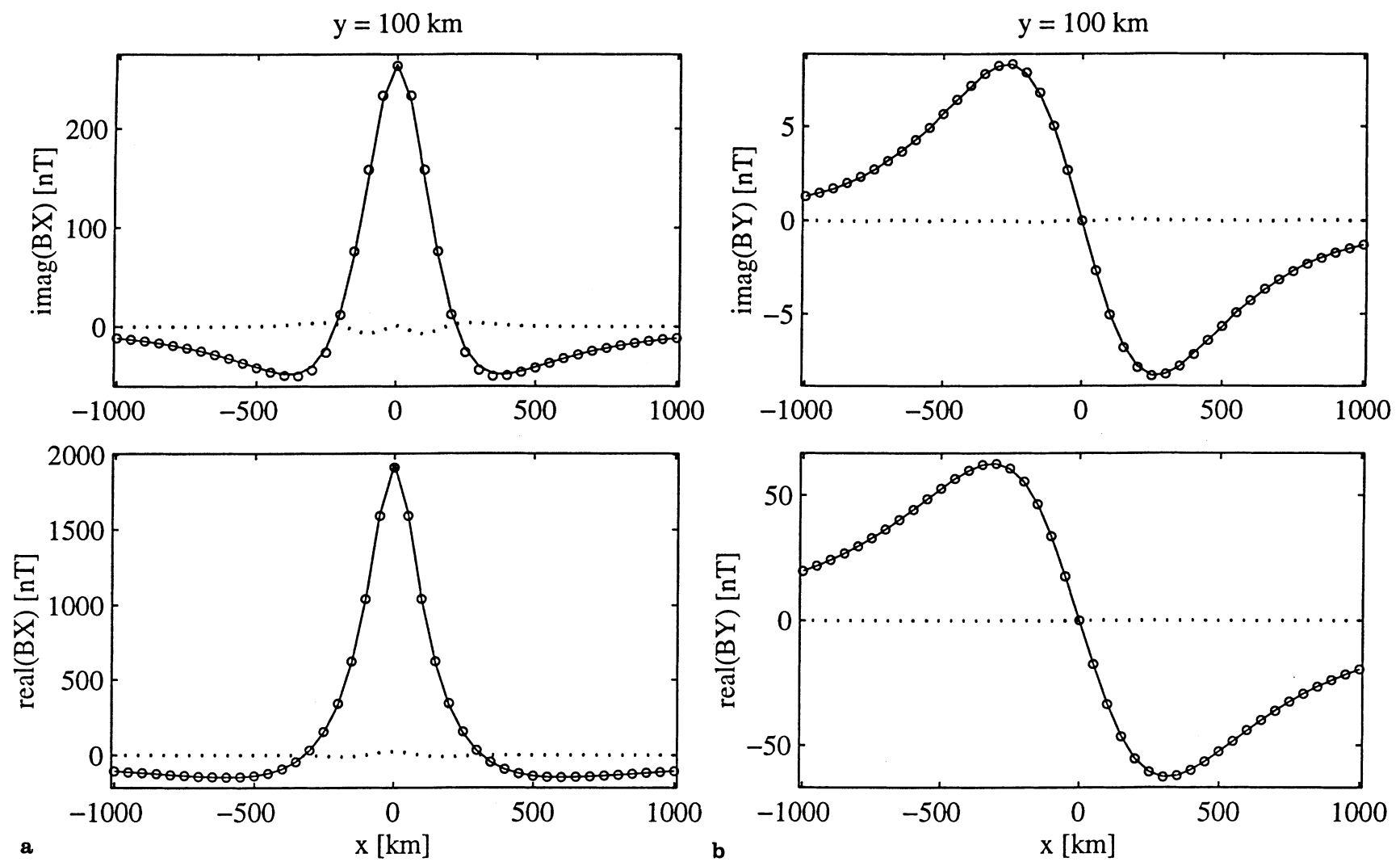

Fig. 3a,b (For continuation see page 1438) 

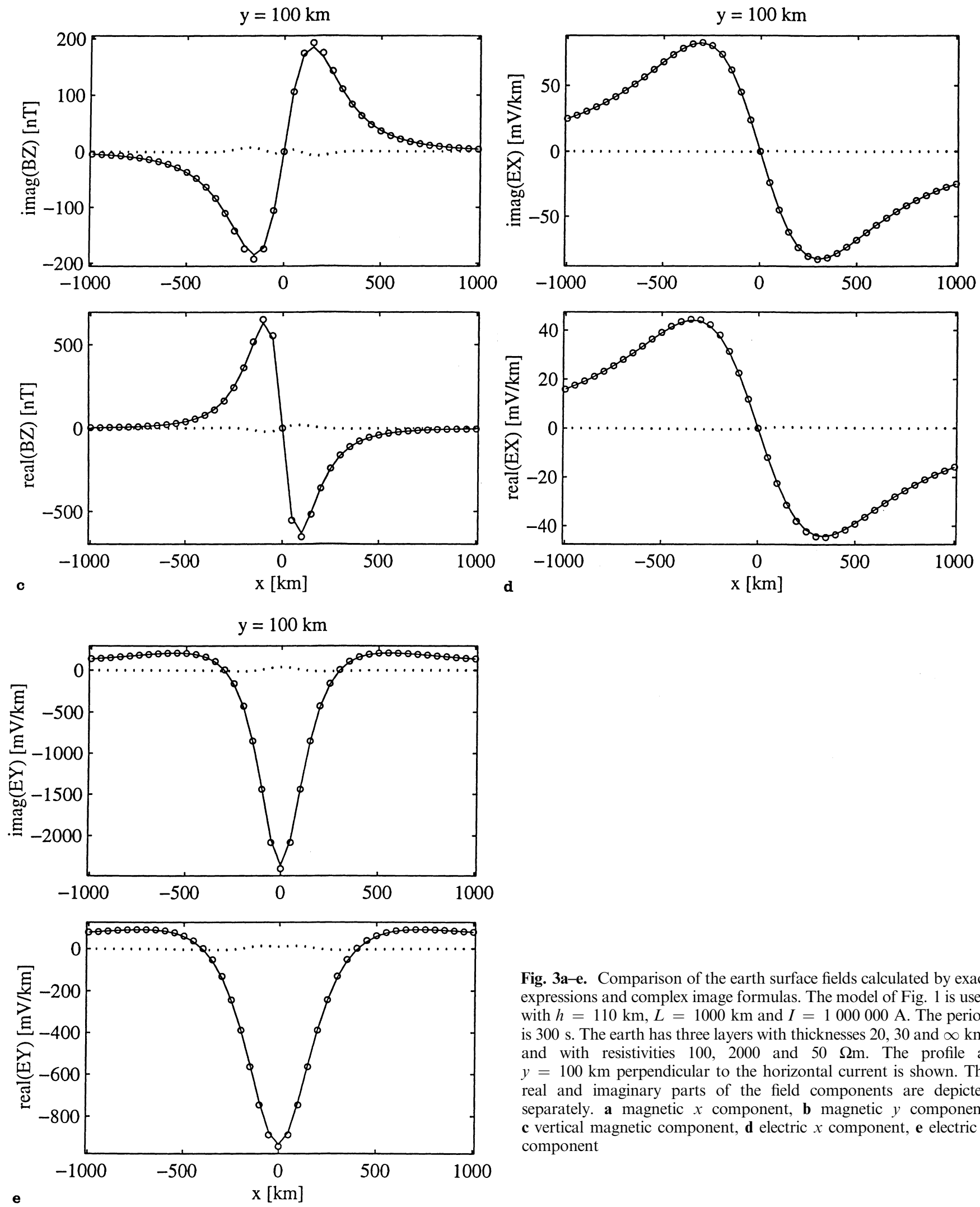

Fig. 3a-e. Comparison of the earth surface fields calculated by exact expressions and complex image formulas. The model of Fig. 1 is used with $h=110 \mathrm{~km}, L=1000 \mathrm{~km}$ and $I=1000000$ A. The period is $300 \mathrm{~s}$. The earth has three layers with thicknesses 20,30 and $\infty \mathrm{km}$, and with resistivities 100,2000 and $50 \Omega \mathrm{m}$. The profile at $y=100 \mathrm{~km}$ perpendicular to the horizontal current is shown. The real and imaginary parts of the field components are depicted separately. a magnetic $x$ component, b magnetic $y$ component, c vertical magnetic component, $\mathbf{d}$ electric $x$ component, e electric $y$ component

imaginary parts of the magnetic field and the real parts of the electric field are totally neglected. Based on the results shown in Figs. 3 and 4, such an approximation is not good enough for all purposes. In fact, the imaginary

parts of the magnetic field and the real parts of the electric field are associated with the time-lag between the primary source above the earth and the secondary currents within the earth. 

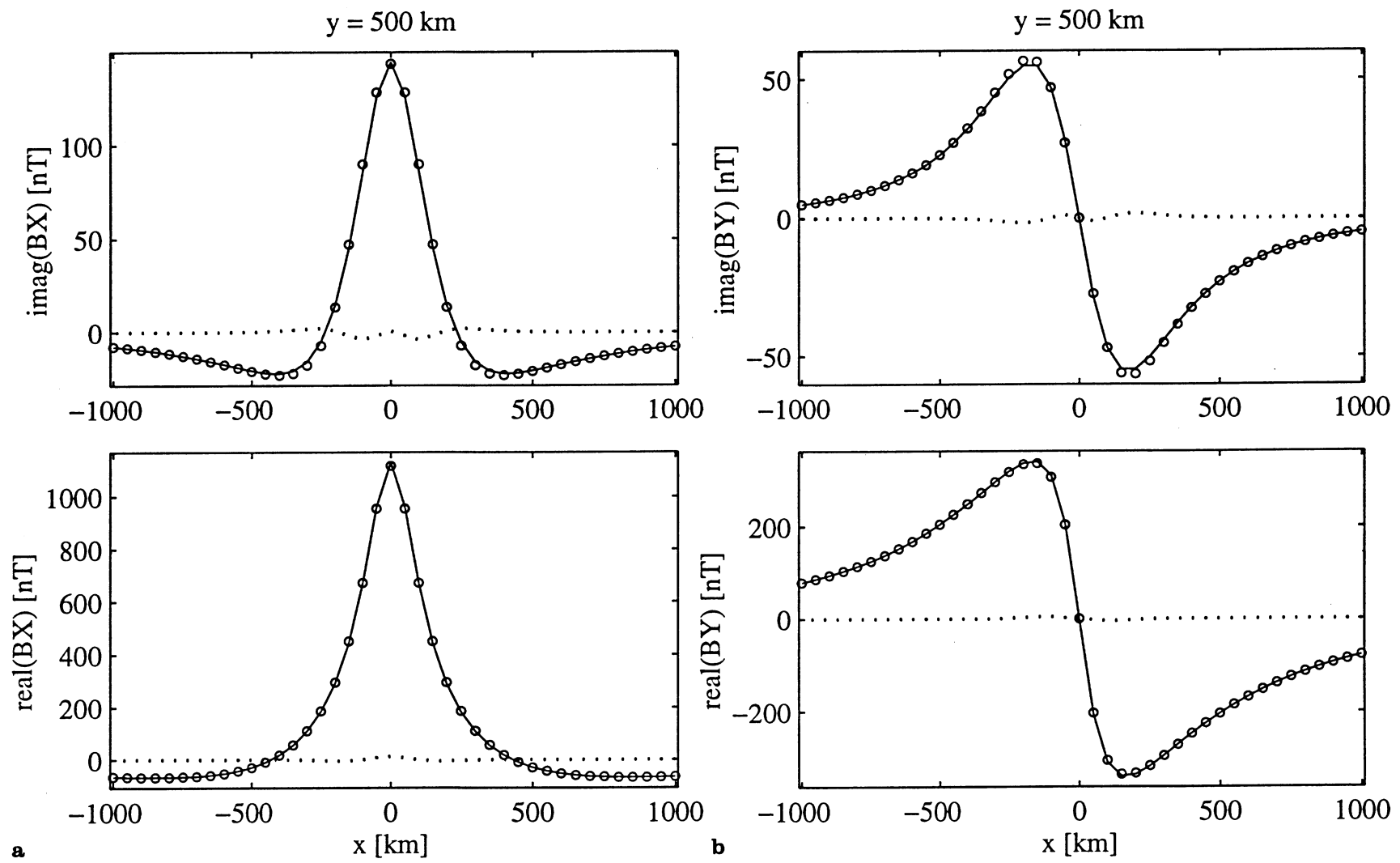

Fig. 4a,b (For continuation please see page 1440)

CIM is thus a very efficient mathematical tool but the complex location of the image source may also be interpreted physically by observing that the real and imaginary parts of the complex skin depth correspond to the central depths of the in-phase and out-of-phase currents flowing in the earth, respectively, (Weidelt, 1972; Szarka and Fischer, 1989).

\section{Concluding remarks}

The complex image method (CIM) is a mathematical technique applicable to problems in which the primary source of an electromagnetic field lies in the vicinity of an interface between two different media, so that the total field is composed of a primary contribution and of a secondary reflected field. In CIM the latter is calculated by replacing the reflecting medium by an image source at a complex location. The ultimate aim is to find convenient closed-form expressions of the total field.

In this paper CIM for calculating the electromagnetic field at the earth's surface due to ionospheric currents is extended to a realistic auroral electrojet of a finite length supplemented by vertical currents at both ends. The theoretical discussion is based on replacing the electrojet system by an equivalent horizontal current distribution for which CIM has been derived before. It is important to note that, as an extension to previous well-known works, the equivalence of the current systems takes account for the induction in the earth and is valid, not only for the magnetic field, but also for the total horizontal electric field at the earth's surface.

The good accuracy of CIM is demonstrated here by comparing numerical results with those obtained by an exact method. The practical significance of CIM results from the fact that CPU times demanded are only a small fraction of those required by exact computations. Thus the complex image technique is extremely applicable to time-critical calculations, such as estimating or predicting geomagnetically induced currents in technological systems.

Although a relatively simple electrojet model is used as an example in this paper, the method can be directly generalized to more complicated systems. A natural approach is to construct the horizontal ionospheric current of short straight filaments. Vertical currents must then be added to keep the total current density divergence-free. As an immediate extension, time-domain calculations can be performed conveniently applying the fast Fourier transform.

\section{Appendix}

Complex image method for a finite electrojet with vertical currents

We prove here the equivalence between the current systems in Figs. 1 and 2 by showing that together with the induction contributions they produce the same hor- 

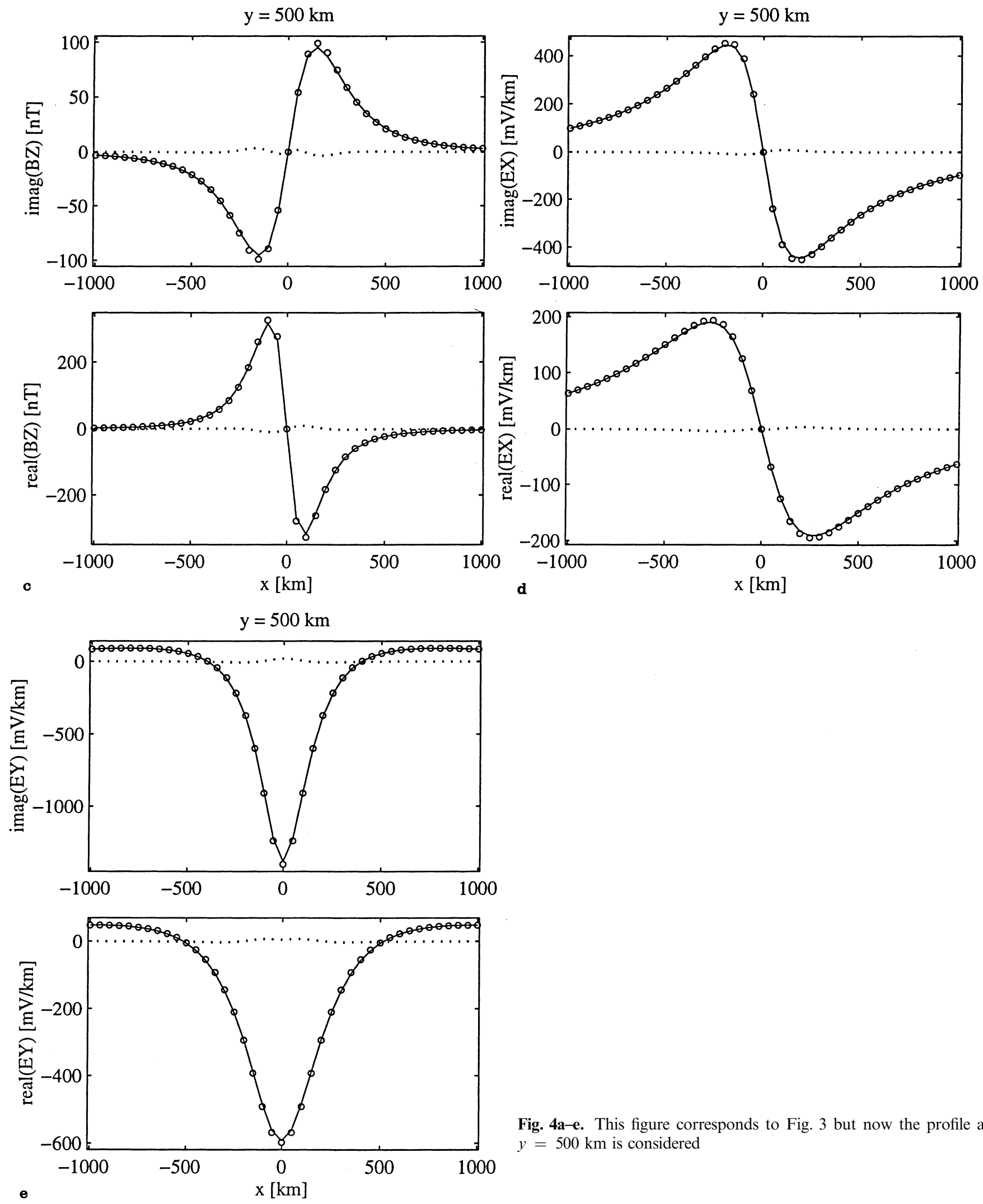

Fig. 4a-e. This figure corresponds to Fig. 3 but now the profile at $y=500 \mathrm{~km}$ is considered

izontal electric field and the same magnetic field at the earth's surface. Then an electrojet of a finite length with vertical currents at its ends can be replaced by a purely horizontal current system, allowing the use of the com- plex image formulation by Thomson and Weaver (1975).

To show the equivalence between Figs. 1 and 2 we consider as an auxiliary result the total electromagnetic 
field produced by the current system shown in Fig. 5 and situated at the height $h$ above the earth's surface (the $x y$ plane of the coordinate system). We prove that the total magnetic field and the total horizontal electric field are zero at the earth's surface (with the accuracy of neglecting the displacement currents). This then justifies the full equivalence of the current systems in Figs. 1 and 2.

For simplicity, assume that the earth is uniform. The possibility of generalizing the results to any layered structure is evident. Using cylindrical coordinates $(\rho, \phi$, $z$ ) with the $z$-axis parallel and coinciding with the downward current, it is clear that the fields are independent of the $\phi$ coordinate. Then the Maxwell equations imply that the triplets $\left(E_{\phi}, B_{\rho}, B_{z}\right)$ and $\left(E_{\rho}, E_{z}\right.$, $\left.B_{\phi}\right)$ are independent of each other. The latter components give the electromagnetic field in the present problem. We assume the time-dependence $e^{i \omega t}$ and denote the permeability, permitivity and conductivity by $\mu, \varepsilon$ and $\sigma$.

It follows from the Maxwell equations that outside the primary sources the magnetic field satisfies:

$\frac{\partial}{\partial \rho}\left(\frac{1}{\rho} \frac{\partial\left(\rho B_{\phi}\right)}{\partial \rho}\right)+\frac{\partial^{2} B_{\phi}}{\partial z^{2}}+k^{2} B_{\phi}=0$,

where the wave number is defined by

$k^{2}=\omega^{2} \mu \varepsilon-i \omega \mu \sigma,-\frac{\pi}{4} \leq \arg k \leq 0$.

When $B_{\phi}$ is known, the electric field is obtained from Ampère's law

$\mu(\sigma+i \omega \varepsilon) E_{\rho}=-\frac{\partial B_{\phi}}{\partial z}$,

$\mu(\sigma+i \omega \varepsilon) E_{z}=\frac{1}{\rho} \frac{\partial\left(\rho B_{\phi}\right)}{\partial \rho}$.

Considering $\mu, \varepsilon$ and $\sigma$ as earth parameters, the field within the earth $(z>0)$ can be calculated from Eqs. (A1)-(A4).

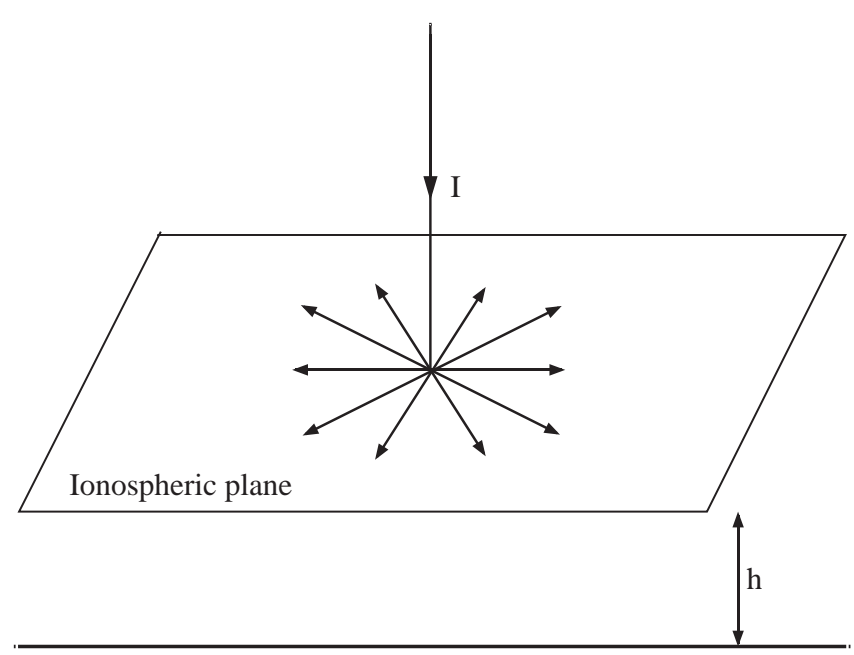

Earth

Fig. 5. Current system consisting of a downward vertical current and a radial horizontal current distribution at the height $h$ above the earth's surface
Equation (A1) is solved by the standard method of the separation of variables. A physically acceptable solution in the earth $(z>0)$ is

$B_{\phi}=\int_{0}^{\infty} D(b) J_{1}(b \rho) e^{-\kappa z} \mathrm{~d} b$,

where

$\kappa^{2}=b^{2}-k^{2},-\frac{\pi}{2}<\arg \kappa \leq \frac{\pi}{2}$

and $J_{l}$ is the Bessel function.

Equations (A3) and (A4) yield

$E_{\rho}=-\frac{i \omega}{k^{2}} \int_{0}^{\infty} \kappa D(b) J_{1}(b \rho) e^{-\kappa z} \mathrm{~d} b$,

$E_{z}=-\frac{i \omega}{k^{2}} \int_{0}^{\infty} b D(b) J_{0}(b \rho) e^{-\kappa z} \mathrm{~d} b$,

where Eq. (A2) and the relation $\mathrm{d} J_{v}(\alpha) / \mathrm{d} \alpha$ $+(v / \alpha) J_{v}(\alpha)=J_{v-1}(\alpha)$ were applied.

The secondary field in the air $(z<0)$ due to earth currents and charges can be calculated exactly in the same way by substituting the air parameters $\mu_{0}, \varepsilon_{0}, \sigma_{0}, k_{0}$ and $\kappa_{0}$ for $\mu, \varepsilon, \sigma, k$ and $\kappa$. The only difference compared to the preceding calculation is that we now must choose the $z$-dependence $e^{\kappa_{0} z}$. Hence

$B_{\phi}=\int_{0}^{\infty} C(b) J_{1}(b \rho) e^{\kappa_{0} z} \mathrm{~d} b$,

$E_{\rho}=\frac{i \omega}{k_{0}^{2}} \int_{0}^{\infty} \kappa_{0} C(b) J_{1}(b \rho) e^{\kappa_{0} z} \mathrm{~d} b$,

$E_{z}=-\frac{i \omega}{k_{0}^{2}} \int_{0}^{\infty} b C(b) J_{0}(b \rho) e^{\kappa_{0} z} \mathrm{~d} b$.

Equations (A5)-(A11) take exact account for the displacement currents generally ignorable in geomagnetic induction studies.

To apply boundary conditions at the earth's surface $(z=0)$, we also need expressions of the primary field. Because there are no charges associated with the current both the electric and the magnetic field are expressible in terms of the vector potential $\boldsymbol{A}=\boldsymbol{A}(\boldsymbol{r}, t)$ :

$\boldsymbol{E}=-\frac{\partial \boldsymbol{A}}{\partial t}=-i \omega \boldsymbol{A}$

$\boldsymbol{B}=\nabla \times \boldsymbol{A}$.

The vector potential can be calculated using a retarded integral over the current density (Stratton, 1941, p. 428). However, to simplify the calculation, we neglect the retardation, i.e. ignore the displacement currents. Then 
$\boldsymbol{A}(\boldsymbol{r}, t)=\frac{\mu_{0}}{4 \pi} \mathrm{e}^{i \omega \mathbf{t}} \int \frac{\boldsymbol{j}\left(\boldsymbol{r}^{\prime}\right)}{R} \mathrm{~d} V^{\prime}$

where $R=\left|\boldsymbol{r}-\boldsymbol{r}^{\prime}\right|, \boldsymbol{j}=\boldsymbol{j}_{h}+\boldsymbol{j}_{v}$ and

$\boldsymbol{j}_{h}=\frac{I}{2 \pi \rho} \delta(z+h) \hat{\boldsymbol{e}}_{\rho}$,

$\boldsymbol{j}_{v}=I \delta(x) \delta(y)(1-\Theta(z+h)) \hat{\boldsymbol{e}}_{z}$.

The total current is denoted by $I$, and $\delta$ and $\Theta$ are the Dirac delta and Heaviside step functions, respectively, and the unit vectors are denoted by $\hat{\boldsymbol{e}}$.

Applying Gradshteyn and Ryzhik (1980, p. 81, Eq. 2.261), we obtain for the vertical component of $\boldsymbol{A}$

$\boldsymbol{A}_{v}=-\frac{\mu_{0} I}{4 \pi} \log \left(\sqrt{\rho^{2}+(z+h)^{2}}+(z+h)\right) \hat{\boldsymbol{e}}_{z}$.

Formally $\boldsymbol{A}_{\boldsymbol{v}}$ also contains another term proportional to $\log (\infty)$, which is a result of neglecting retardation, while on the other hand the current is allowed to continue to infinity. This conclusion is supported by noting that the electric field has a logarithmic spatial dependence also in the case of an infinite line current if the retardation is ignored. Then in the exact formula the logarithm is replaced by a Hankel function having a proper behaviour for large arguments (e.g. Pirjola, 1982, p. 36). Regarding the magnetic field, a constant in the vector potential plays no role. Another justification for the omission of $\log (\infty)$ is obtained because the complex image we are aiming at in this paper would also produce a logarithmic infinity evidently cancelling that due to the primary current.

A substitution of Eq. (A15) into Eq. (A14) yields for the horizontal component of $\boldsymbol{A}$

$$
\begin{aligned}
\boldsymbol{A}_{h}= & \frac{\mu_{0} I}{8 \pi^{2}} \int_{0}^{2 \pi}\left(\hat{\hat{\mathbf{e}}_{\mathbf{y}}} \cos \varphi+\hat{\mathbf{e}}_{\mathbf{x}} \sin \varphi\right) \mathrm{d} \varphi \\
& \times \int_{0}^{\infty} \frac{\mathrm{d} \rho^{\prime}}{\sqrt{\left(x-\rho^{\prime} \sin \varphi\right)^{2}+\left(y-\rho^{\prime} \cos \varphi\right)^{2}+(z+h)^{2}}},
\end{aligned}
$$

where $\varphi$ is just a variable of integration not equal to the cylindrical coordinate $\phi$. The integration over $\rho^{\prime}$ can be performed applying Gradshteyn and Ryzhik (1980, p. 81 , Eq. 2.261), ignoring again a $\log (\infty)$ term. Consequently,

$$
\boldsymbol{A}_{h}=-\frac{\mu_{0} I}{8 \pi^{2}}\left(\hat{\boldsymbol{e}}_{y} \int_{0}^{2 \pi} \cos \varphi \log (r-x \sin \varphi-y \cos \varphi) \mathrm{d} \varphi\right.
$$

$$
\left.+\hat{\mathbf{e}}_{x} \int_{0}^{2 \pi} \sin \varphi \log (r-x \sin \varphi-y \cos \varphi) \mathrm{d} \varphi\right),
$$

where $r=\sqrt{x^{2}+y^{2}+(z+h)^{2}}$. The integrals Eq. (A19) can be modified by integration by parts:

$$
\begin{gathered}
\int_{0}^{2 \pi} \cos \varphi \log (r-x \sin \varphi-y \cos \varphi) \mathrm{d} \varphi \\
=-\int_{0}^{2 \pi} \frac{y \sin ^{2} \varphi-x \sin \varphi \cos \varphi}{r-x \sin \varphi-y \cos \varphi} \mathrm{d} \varphi, \\
\int_{0}^{2 \pi} \sin \varphi \log (r-x \sin \varphi-y \cos \varphi) \mathrm{d} \varphi \\
=-\int_{0}^{2 \pi} \frac{x \cos ^{2} \varphi-y \sin \varphi \cos \varphi}{r-x \sin \varphi-y \cos \varphi} \mathrm{d} \varphi .
\end{gathered}
$$

The right-hand sides of Eq. (A20) and (A21) can be evaluated by the calculus of residues (Arfken, 1985, pp. 400-421), and after tedious work the final result is

$$
\begin{aligned}
\boldsymbol{A}_{h} & =\frac{\mu_{0} I}{4 \pi} \frac{\sqrt{\rho^{2}+(z+h)^{2}}-|z+h|}{\rho} \hat{\boldsymbol{e}}_{\rho} \\
& =\frac{\mu_{0} I}{4 \pi} \frac{\rho}{\sqrt{\rho^{2}+(z+h)^{2}}+|z+h|} \hat{\boldsymbol{e}}_{\rho} .
\end{aligned}
$$

Applying Eqs. (A13), (A17) and (A22) it is seen that the current system shown in Fig. 5 produces no primary magnetic field for $z>-h$. (cf. Fukushima, 1976).

The primary electric field is obtained from Eqs. (A12), (A17) and (A22):

$$
\begin{aligned}
& E_{\rho}=-\frac{i \omega \mu_{0} I}{4 \pi} \frac{\sqrt{\rho^{2}+(z+h)^{2}}-|z+h|}{\rho}, \\
& E_{z}=\frac{i \omega \mu_{0} I}{4 \pi} \log \left(\sqrt{\rho^{2}+(z+h)^{2}}+(z+h)\right) .
\end{aligned}
$$

In Eq. (A24) the logarithm is taken of a number having a unit of length [as in Eq. (A17)]. This difficulty, which would require a more detailed examination, follows from the neglect of the retardation. However, because we do not need $E_{z}$, it will not be considered further here.

$E_{\rho}$ and $B_{\phi}$ are continuous across the earth's surface. Thus, from Eqs. (A5), (A7), (A9), (A10) and (A23) we obtain at $z=0$ :

$$
\begin{gathered}
-\frac{i \omega \mu_{0} I}{4 \pi} \frac{\sqrt{\rho^{2}+h^{2}}-h}{\rho}+\frac{i \omega}{k_{0}^{2}} \int_{0}^{\infty} \kappa_{0} C(b) J_{1}(b \rho) \mathrm{d} b \\
=-\frac{i \omega}{k^{2}} \int_{0}^{\infty} \kappa D(b) J_{1}(b \rho) \mathrm{d} b, \\
\int_{0}^{\infty} C(b) J_{1}(b \rho) \mathrm{d} b=\int_{0}^{\infty} D(b) J_{1}(b \rho) \mathrm{d} b .
\end{gathered}
$$

in Multiply Eq. (A26) by $\rho J_{1}\left(b^{\prime} \rho\right),\left(b^{\prime}>0\right)$, and integrate both sides over $\rho$ from 0 to $\infty$. The use of 


$$
\int_{0}^{\infty} J_{v}(p q) J_{v}\left(p^{\prime} q\right) q \mathrm{~d} q=\frac{\delta\left(p-p^{\prime}\right)}{p},
$$

where $v>-1 / 2$, and $p$ and $p^{\prime}$ are real (Arfken, 1985, p. 594), shows that $C(b)=D(b)$. We may now substitute $C(b)$ for $D(b)$ in Eq. (A25), multiply by $\rho J_{1}\left(b^{\prime} \rho\right)$, and integrate over $\rho$. Applying Eq. (A27) we obtain

$$
\frac{i \omega}{b^{\prime}}\left(\frac{\kappa_{0}}{k_{0}^{2}}+\frac{\kappa}{k^{2}}\right) C\left(b^{\prime}\right)=\frac{i \omega \mu_{0} I}{4 \pi} \int_{0}^{\infty} J_{1}\left(b^{\prime} \rho\right)\left(\sqrt{\rho^{2}+h^{2}}-h\right) \mathrm{d} \rho .
$$

The term resulting from $-h J_{1}\left(b^{\prime} \rho\right)$ on the right-hand side of Eq. (A28) is integrated using Gradshteyn and Ryzhik (1980, p. 665, Eq. 6.511.1). The other term is integrated by parts noting that $J_{1}$ is the negative derivative of $J_{0}$. The substitution of the upper limit $\rho$ $=\infty$ gives an indefinite value but, as in the case of logarithmic infinities above, we ignore it by considering it to be caused by the neglect of the retardation. This procedure leads to an integral that can be calculated using Gradshteyn and Ryzhik (1980, p. 682, Eq. 6.554.1), and Eq. (A28) yields

$D\left(b^{\prime}\right)=C\left(b^{\prime}\right)=\frac{\mu_{0} I}{4 \pi} k_{0}^{2} k^{2} \frac{e^{-h b^{\prime}}}{b^{\prime}\left(k^{2} \kappa_{0}+k_{0}^{2} \kappa\right)}$.

Equations (A5)-(A11) and (A23) now permit the determination of the electromagnetic field at any point below the ionosphere $(z>-h)$. At the earth's surface

$E_{\rho}=-\frac{i \omega \mu_{0} I}{4 \pi} \int_{0}^{\infty} \frac{k_{0}^{2} \kappa e^{-h b}}{b\left(k^{2} \kappa_{0}+k_{0}^{2} \kappa\right)} J_{1}(b \rho) \mathrm{d} b$

$B_{\phi}=\frac{\mu_{0} I}{4 \pi h} \int_{0}^{\infty} \frac{h k_{0}^{2} k^{2} e^{-h b}}{b\left(k^{2} \kappa_{0}+k_{0}^{2} \kappa\right)} J_{1}(b \rho) \mathrm{d} b$.

Equations (A30) and (A31), in which the integrals are dimensionless, show that the total magnetic and horizontal electric field due to the current system of Fig. 5 are not strictly zero. In both equations the integrands are proportional to the square of the propagation constant $k_{0}$ of the air, whose setting equal to zero is equivalent to the neglecting the displacement currents or retardation. This indicates that the integrands are small, making the values of the integrals, and consequently $E_{\rho}$ and $B_{\phi}$, also small. However, a proof would require a more careful analysis of the integrals, which we did numerically, and we found that

$B_{\phi} \ll \mu_{0} I / h$ and $E_{\rho} \ll \omega \mu_{0} I$,

where the ratios between the left-hand and right-hand sides of these inequalities are roughly speaking in the order of $\left(k_{0} / k\right)^{2}$ implying that $E_{\rho}$ and $B_{\phi}$, are zero in practice.

We also made a numerical test in which a current system similar to that shown in Fig. 5 was constructed of thirty "U"-shaped electrojet systems of the type presented in Fig. 1. One end of each electrojet was at $(0$, $0,-h)$ and the other end lay on a horizontal circle at $12^{\circ}$ separation. Thus, one of the field-aligned currents of each " $U$ "-shaped system coincided forming the vertical current in Fig. 5. Having the point of observation close to the origin, the other field-aligned currents did not have an essential effect. For the earth structure we used the "Central Finland Model" consisting of six layers (Viljanen et al., 1993). The total current intensity was 1 MA, the height and the length of the electrojets were 110 and $1500 \mathrm{~km}$, and the point of observation was located at the earth's surface at a distance of about $150 \mathrm{~km}$ from the origin. The period considered was $60 \mathrm{~s}$. The electromagnetic field was calculated exactly, and it was found that the horizontal electric field was very small, a few $\mathrm{mV} / \mathrm{km}$, and the magnetic field was also extremely small, about $1 \mathrm{nT}$. This supports the conclusion that the current system depicted in Fig. 5 does not give rise to (practically) any horizontal electric or any magnetic field at the earth's surface.

Because the current system of Fig. 5 creates no magnetic or horizontal electric field it can be added to any current system without affecting these fields. Doing this at both ends of the electrojet presented in Fig. 1 leads to the system shown in Fig. 2. Thus we have arrived at an equivalent purely horizontal current distribution that may be substituted for a finite electrojet supplemented by vertical field-aligned currents (as far as we are not interested in the vertical electric field component).

Acknowledgements. Topical Editor D. Alcaydé thanks A. Agarwal and N. Olsen for their help in evaluating this paper.

\section{References}

Amm, O., Direct determination of the local ionospheric Hall conductance distribution from two-dimensional electric and magnetic field data: application of the method using models of typical ionospheric electrodynamic situations. J. Geophys. Res., 100, 21473-21488, 1995.

Amm, O., Ionospheric elementary current systems in spherical coordinates and their application J. Geomagn. Geolectr., 49, 947-955, 1997.

Arfken, G., Mathematical methods for physicists, 3 Edn., Academic Press, New York, 1985.

Bannister, P. R., Applications of complex image theory, Radio Sci., 21, 605-616, 1986.

Boteler, D. H., and R. J. Pirjola, The complex-image method for calculating the magnetic and electric fields produced at the surface of the Earth by the auroral electrojet, Geophys. J. Int., 132, 1, 31-40, 1998.

Boteler, D. H., R. J. Pirjola, and H. Nevanlinna, The effects of geomagnetic disturbances on electrical systems at the earth's surface, Adv. Space Res., in press, 1998.

Fukushima, N., Generalized theorem for no ground magnetic effect of vertical currents connected with Pedersen currents in the uniform-conductivity ionosphere, Rep. Ionosphere Space Res. Jpn., 30, 1/2, 35-40, 1976.

Gradshteyn, I. S., and I. W. Ryzhik, Table of integrals, series, and products, Academic Press, New York, 1980.

Häkkinen, L., and R. Pirjola, Calculation of electric and magnetic fields due to an electrojet current system above a layered earth, Geophysica, 22, 31-44, 1986 
Lanzerotti, L. J., and G. P. Gregori, Telluric currents: the natural environment and interactions with man-made systems, the earth's electrical environment, in Studies in geophysics, Eds. E. P. Krider, R. G. Roble, and T. M. Usselman, National Academy Press, Washington, D.C., USA, pp., 232257, 1986.

Lühr, H., N. Klöcker, and S. Thürey, Ground-based observations of a very intense substorm-related pulsation event, J. Geophys., 55, 41-53, 1984.

Mogilatov, V., Excitation of a half-space by a radial current sheet source, Pure Appl. Geophys. (PAGEOPH), 147, 763-775, 1996.

Petschek, H. E., and W. E. Feero, Workshop focuses on space weather's impact on electric power, EOS Trans., American Geophysical Union, 78, 217-218, 1997.

Pirjola, R., Electromagnetic induction in the earth by a plane wave or by fields of line currents harmonic in time and space, Geophysica, 18, 1-161, 1982.

Pirjola, R. J., and L. V. T. Häkkinen, Electromagnetic field caused by an auroral electrojet current system model, In: Environmental and space electromagnetics, Ed. H. Kikuchi, Springer, Berlin, Heidelberg, New York, pp. 288-298, 1991.

Pirjola, R., and A. Viljanen, On geomagnetically induced currents in the Finnish $400-\mathrm{kV}$ power system by an auroral electrojet current, IEEE Trans. power delivery, 4, 1239-1245, 1989.

Schmucker, U., Anomalies of geomagnetic variations in the southwestern United States, Bull. Scripps Inst. Ocean., 13,
University of California Press, Berkeley and Los Angeles, California, USA, 1970.

Stratton, J. A., Electromagnetic theory, McGraw-Hill, New York, 1941.

Szarka, L., and G. Fischer, Electromagnetic parameters at the surface of a conductive halfspace in terms of the subsurface current distribution, Geophys. Trans., 25, 157-172, 1989.

Thomson, D. J., and J. T. Weaver, The complex image approximation for induction in a multilayered Earth, J. Geophys. Res., 80, 123-129, 1975.

Viljanen, A., The relation between geomagnetic variations and their time derivatives and implications for estimation of induction risks, Geophys. Res. Lett., 24, 631-634, 1997.

Viljanen, A., R. Pirjola, and L. Häkkinen, An attempt to reduce induction source effects at high latitudes, J. Geomagn. Geoelectr., 45, 817-831, 1993.

Wait, J. R., Wave propagation theory, Pergamon Press New York, 1981.

Wait, J. R., Comment on 'Excitation of a half-space by a radial current sheet source' by V. Mogilatov, Pure Appl. Geophys. (PAGEOPH), 150, 155, 1997.

Wait, J. R., and K. P. Spies, On the representation of the quasistatic fields of a line current source above the ground, Can. J. Phys., 27, 2731-2733, 1969.

Weidelt, P., The inverse problem of geomagnetic induction, Z. Geophys., 38, 257-289, 1972. 